\title{
Observations and discoveries of supernova remnants with GMRT
}

\author{
Subhashis Roy ${ }^{1}$ and Sabyasachi Pal ${ }^{2,3}$ \\ ${ }^{1}$ NCRA-TIFR, Pune University Campus, \\ Pune-411007, India \\ email: roy@ncra.tifr.res.in \\ ${ }^{2}$ Indian Centre for Space Physics, \\ Kolkata-700084, India \\ ${ }^{3}$ Ionospheric and Earthquake Research Centre, \\ Kolkata-7211154, India \\ email: sabya@csp.res.in
}

\begin{abstract}
We have measured HI absorption distance to the youngest Galactic supernova remnant G1.9+0.3. Absorption by known anomalous velocity features near the Galactic centre (GC) puts a lower limit on its distance from Sun as $10 \mathrm{kpc}, 2 \mathrm{kpc}$ further away from the GC. We have found a small diameter $\left(1.6^{\prime}\right)$ shell like structure G354.4+0.0, that shows polarised emission in the NVSS. Based on its morphology, angular size, HI distance and its spectrum between 1.4 $\mathrm{GHz}$ and $330 \mathrm{MHz}$, it is perhaps the second youngest SNR in the Galaxy that is expanding in a dense environment of an HII region surrounding it. Our pilot observation of the inner Galactic 4 th quadrant within $337^{\circ}<l<354^{\circ}$ with a fixed Galactic latitude of $0.37^{\circ}$ has confirmed G345.1-0.2 as an SNR.
\end{abstract}

Keywords. supernova remnants, radio continuum: ISM

\section{Introduction}

The number of supernova remnants (SNRs) in our Galaxy is catalogued to be 273 (Green 2009). However, the expected number of SNRs based on the OB star counts, pulsar birth rates and Iron abundance in our Galaxy, and supernova rates in Local Group galaxies is 1000 (Tammann et al. 1994). This deficit is likely due to the result of instrumental insensitivity towards old, faint, large remnants and the effect of poor angular resolution working against detection of small diameter SNRs in low resolution surveys(Green 1991). A lack of their detection would otherwise indicate our inability to characterise the stellar evolution in our own Galaxy or in the prediction of the timescale over which an SNR remains detectable in the ISM. The missing remnants are likely concentrated towards the inner Galaxy, where the diffuse emission causes the most confusion.

Given the expected supernova rate in the Galaxy of 2.8 per century (Li et al. 2011) we expect to see about 20 supernovae remnants of age less than 700 years, but only 4 are known (Tycho, Kepler, Cas A and G1.9+0.3). Among these four SNRs, G1.9+0.3 is the smallest in angular size $\sim 1.2^{\prime}$ (Green \& Gull 1984). This indicated it to be comparatively young, and remarkably enough, its size measured from Chandra X-ray image was found to be larger than measured from VLA radio data taken two decades back and from its expansion over the last two decades it has been shown to be the youngest known Galactic supernova remnant of age about 150 years (Reynolds et al. 2008). Based on very high absorbing column density found from their X-ray observations, Reynolds et al. (2008) suggested it to be located at the distance of the Galactic centre (GC) of $8 \mathrm{kpc}$. This distance is, however, a lower limit, as its location beyond the GC would not change the 
absorption column density much. Given that its physical properties like linear size and expansion velocity depends on conversion of angular size to linear size depending on its distance, a more direct determination of its distance is important. We carried out HI absorption measurements towards it in 2004. In the next section, we present the results and determine its distance and discuss its consequent ramifications on its properties.

Most of the missing remnants in the Galaxy are likely concentrated towards the inner Galaxy. Therefore, we had used GMRT at $330 \mathrm{MHz}$ to confirm candidate SNRs from Molonglo observatory synthesis telescope (MOST) survey of the GC region (Roy \& Bhatnagar 2006). The main objective of these observations was to confirm the nature of certain candidate SNRs. In one of these fields, we identified a small shell like structure G354.4+0.0 that was seen just outside the primary beam of that field. In the next section, we describe its confirmation as a newly discovered young SNR in the Galaxy.

A histogram plot of the number of SNRs in the Galaxy (Green 2009) as a function of Galactic longitude peaks within $|l| \sim 0^{\circ}-20^{\circ}$. The number of SNRs catalogued within $0^{\circ}<l<20^{\circ}$ is 65 , and between $340^{\circ}<l<360^{\circ}$ is 42 , indicating a clear asymmetry in SNR distribution between the 1st and 4th quadrant of the inner Galaxy. This is mostly due to the new discovery of SNRs by Brogan et al. (2006) within $4.5^{\circ}<l<22^{\circ}$ and $|b|<1.5^{\circ}$ region of the Galaxy. Asymmetry in supernova rate or in the timescale for which SNRs remain detectable in the ISM are unlikely to be different from the 1st to the 4th quadrant of the Galaxy. Therefore, observations of the inner 4th quadrant of the Galaxy with the sensitivity and resolution attained by Brogan et al. (2006), will discover missing remnants $(\sim 23)$ in the Galactic 4th quadrant. Earlier observations of this region has been carried out by the Molonglo Observatory Synthesis Telescope (MOST) at 843 $\mathrm{MHz}$ and claimed to have reached a sensitivity of $2 \mathrm{mJy} \cdot \mathrm{beam}^{-1}$ (Whiteoak \& Green 1996). Assuming a spectral index of -0.5 for the Galactic SNRs, their sensitivity was actually 1.5 times more than Brogan et al. (2006) (both had similar resolution). This suggests that VLA observations of SNRs in the inner 1st quadrant of the Galaxy to have better dynamic range than the MOST survey. Since GMRT has high sensitivity at $330 \mathrm{MHz}$, and is sensitive to structures with angular scales of $\sim 30^{\prime}$, we have carried out a pilot survey of this region with $337^{\circ}<l<354^{\circ}$ with a fixed Galactic latitude of $0.37^{\circ}$, with a plan to extend this survey in future. In the next section, we describe the outcomes.

\section{Results and Discussions}

\subsection{HI absorption towards G1.9+0.3}

Fig. 1 shows the HI absorption spectrum towards the source. Since the direction of the object is close to that of the GC, Galactic rotation cannot be used to constrain its distance. However, absorption by the anomalous velocity features, whose velocities and distance from GC are known could be used to constrain its distance. In the spectrum, absorption near $0 \mathrm{~km} \cdot \mathrm{sec}^{-1}$ is believed to be from local gas seen nearly perpendicular to its Galactic rotation direction. Prominent absorption is seen near velocity of -50 $\mathrm{km} \cdot \mathrm{sec}^{-1}$. Weak absorption is also seen near velocity of $150 \mathrm{~km} \cdot \mathrm{sec}^{-1}$. The 3 - $\mathrm{kpc}$ arm located about $5 \mathrm{kpc}$ away from Sun is known to have a velocity of about $-50 \mathrm{~km} . \mathrm{sec}^{-1}$ at this Galactic longitude. The absorption near $150 \mathrm{~km} \cdot \mathrm{sec}^{-1}$ is believed to be caused by the 'Feature-I' located $2 \mathrm{kpc}$ further from the GC (Cohen 1975). Therefore, lower limit on the distance to G1.9+0.3 is $10 \mathrm{kpc}$ from Sun. Consequently, its diameter is modified to $>3.5 \mathrm{pc}$, and mean expansion velocity to $11,000 \mathrm{~km} \cdot \mathrm{sec}^{-1}$. Assuming this SNR to have been created by a Type Ia explosion, Ksenofontov et al. (2010) calculated its nonthermal properties and found its $\mathrm{TeV}$ gamma ray energy to depend on its (distance) ${ }^{-11}$. If located 


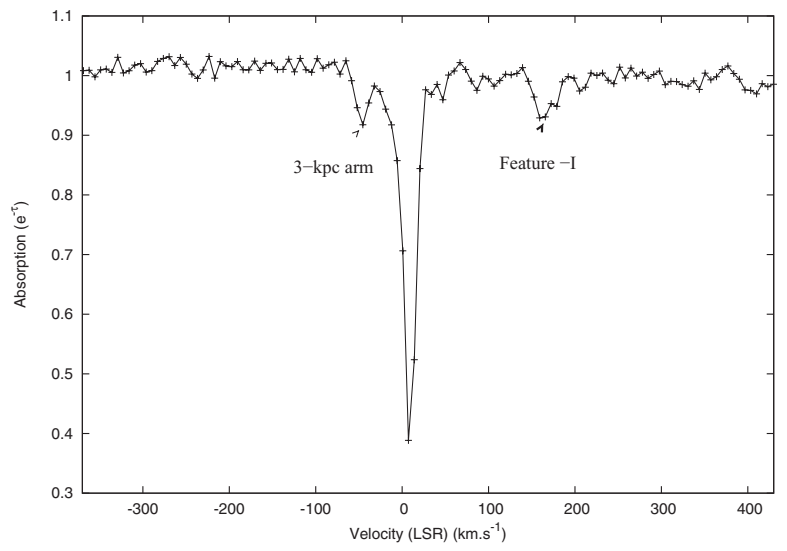

Figure 1. HI absorption spectrum towards the youngest SNR G1.9+0.3.

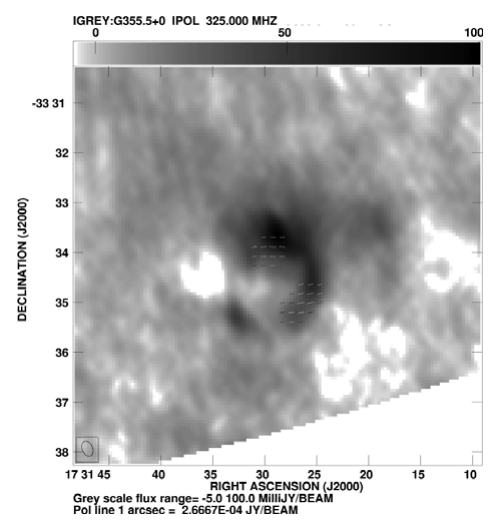

Figure 2. A $325 \mathrm{MHz}$ image of G354.4+0.0. Polarisation vectors from NVSS map are overlaid. Resolution $\sim 15^{\prime \prime}$, rms noise 3 mJy.beam ${ }^{-1}$.

within $5.6 \mathrm{kpc}$, they predicted its $\mathrm{TeV}$ gamma rays to be detectable by future instruments like Cerenkov telescope array. However, our results rule out any such possible detection in future.

\subsection{Discovery of an young SNR five degrees away from the $G C$}

The continuum map of the source G354.4+0.0 made from $\mathrm{P}$ band data having a resolution of $\sim 15^{\prime \prime}$ is shown in Fig. 2. This map is sensitive to large scale structures up to $30^{\prime}$. The object shows morphology of a partial shell, and the size of it as measured by taking several cross-cuts across it and then finding their average is $1.6^{\prime}$. We have searched the NRAO VLA sky survey (Condon et al. 1998) to detect any polarized emission from the partial shell structure and thereby confirm non-thermal emission from it. Polarization vectors from NVSS are overlaid on Fig. 2. To make the polarization total intensity and angle images from the NVSS Stokes Q and U maps ( $\mathrm{rms}$ noise $\sim 0.3$ mJy.beam ${ }^{-1}$ ), all pixels below a signal to noise ratio of 4.5 were blanked and correction for noise bias in polarized total intensity was made. We do detect significant polarized emission from near the two brightest parts of the shell like structure with peak polarized flux densities of $\sim 2.2$ mJy.beam $^{-1}$ (Fig. 2).

We have also observed this object at $1.42 \mathrm{GHz}$ with GMRT. Based on the flux density of the shell between $330 \mathrm{MHz}$ and $1.4 \mathrm{GHz}$, we find its spectral index to be $0.0 \pm 0.1$. This flat spectral index is unexpected from a shell type SNR. To search for any free-free absorbing screen towards the object, we made a low resolution map from the $\mathrm{L}$ band GMRT data that shows diffuse extended emission of size $\sim 5^{\prime}$ on and around the location of the shell. This diffuse emission has been catalogued by the PMN survey (Wright et al. 1996) at $4.8 \mathrm{GHz}$ (flux density 3.7 Jy). A comparison of the flux density of this extended emission between the above two frequencies also shows a flat spectrum for it, indicating thermal emission from the extended emission. This is also confirmed from its detection in the $60 \mu \mathrm{m}$ IRAS map. The diffuse emission itself contributes $2.7 \mathrm{Jy}$ at $1.4 \mathrm{GHz}$. However, from the same region, the measured flux density due to the diffuse emission only is 0.9 Jy at $330 \mathrm{MHz}$. This shows the thermal extended emission to undergo self absorption at $330 \mathrm{MHz}$ with optical depth $(\tau) \sim 4$. Assuming an intrinsic spectral index of -0.6 $\left(\mathrm{S}(\nu) \propto \nu^{\alpha}\right)$ for the shell, the required $\tau$ for the free-free absorbing medium to flatten the 
observed spectrum of the shell at $330 \mathrm{MHz}$ to zero is about 0.9. Given the $\tau \sim 4$ towards the HII region along the same line of sight, this indicates the shell to be embedded within the HII region.

Presence of non-thermal emission as indicated by the polarised emission and shell morphology indicates it to be a newly discovered SNR. HI absorption spectrum made from the GMRT $1.4 \mathrm{GHz}$ data shows absorption by the 3-kpc arm, indicating it to be located more than $5 \mathrm{kpc}$ from Sun. We believe G354.4+0.0 is likely located at the GC distance of $8 \mathrm{kpc}$. From the angular size and distance, the diameter of the SNR is about $3.7 \mathrm{pc}$. From the emission measure of the extended HII region around it, we estimate the electron density and temperature of the ionised gas to be $70 \mathrm{~cm}^{-3}$ and $\sim 1000 \mathrm{~K}$ respectively. Initial expansion of the supernova with a typical velocity of 10,000 $\mathrm{km} \cdot \mathrm{sec}^{-1}$ in the dense environment of the ionised gas has swept up a mass of about $4 M_{\odot}$. Assuming an initial ejecta mass of $\sim 1 M_{\odot}$ indicates the SNR is presently in adiabatic stage of expansion and its age is about 260 years. This shows G354.4+0.0 could be the second youngest SNR in the Galaxy. More details shall be found in Roy \& Pal (2013).

\subsection{A pilot survey of the inner 4th quadrant of the Galaxy at $330 \mathrm{MHz}$ with GMRT}

Within the region of the survey mentioned in the introduction, we achieved a resolution of about $19^{\prime \prime} \times 13^{\prime \prime}$. The rms noise attained was 6-10 mJy.beam ${ }^{-1}$, a factor of 2-3 higher than expected. We detect the following 9 known SNRs: G337.8-0.1, G338.3-0.0, G340.6+0.3, G341.8-0.2, G344.6-0.1, G346.6-0.2, G348.5+0.0, G348.7+0.3, G351.2+0.1. Whiteoak \& Green (1996) suggested 2 objects of high surface brightness, G337.2+0.1, G339.6-0.6 as candidate SNRs. We could not detect them, and they are unlikely to be SNRs. One of the candidate SNRs G345.1-0.2 described by Whiteoak \& Green (1996) is seen in our $330 \mathrm{MHz}$ maps. Between 330 and $843 \mathrm{MHz}$, its spectral index is found to be steep, -0.6 . Therefore, a shell type morphology and steep spectral index confirms G345.1-0.2 to be an SNR.

\section{Acknowledgement}

We thank the staff of GMRT that made these observations possible. GMRT is run by National Center for Radio Astrophysics of the Tata Institute of Fundamental Research. Work of SP is supported by a grant of MOES.

\section{References}

Brogan, C. L., Gelfand, J. D., Gaensler, B. M., Kassim, N. E., \& Lazio, T. J. W. 2006, ApJL, 639, L25

Cohen, R. J. 1975, MNRAS, 171, 659

Condon, J. J., Cotton, W. D., Greisen, E. W., et al. 1998, AJ, 115, 1693

Green, D. A. 1991, PASP, 103, 209

-. 2009, Bulletin of the Astronomical Society of India, 37, 45

Green, D. A. \& Gull, S. F. 1984, Nature, 312, 527

Ksenofontov, L. T., Völk, H. J., \& Berezhko, E. G. 2010, ApJ, 714, 1187

Li, W., Chornock, R., Leaman, J., et al. 2011, MNRAS, 412, 1473

Reynolds, S. P., Borkowski, K. J., Green, D. A., et al. 2008, ApJL, 680, L41

Roy, S. \& Bhatnagar, S. 2006, Journal of Physics Conference Series, 54, 152

Roy, S. \& Pal, S. 2013, to be submitted to ApJL

Tammann, G. A., Loeffler, W., \& Schroeder, A. 1994, ApJS, 92, 487

Whiteoak, J. B. Z. \& Green, A. J. 1996, AApS, 118, 329

Wright, A. E., Griffith, M. R., Hunt, A. J., et al. 1996, ApJS, 103, 145 


\section{Discussion}

SANKRIT: How many HII regions are found in the survey region?

RoY S.: I am yet to count the number of HII regions in the survey. However the number of HII regions that morphologically appear similar to shell type SNRs are $\sim 10$, quite close to the number of known SNRs in the region. 